\title{
Dynamic Navigation Method with Multisubstations Based on Doppler Shift
}

\author{
Jianyang Zhao, ${ }^{1,2}$ Weihong Ding, ${ }^{3}$ Zujun Ding, ${ }^{3}$ Lianfeng Shen, ${ }^{4}$ and Jin Zhao ${ }^{5}$ \\ ${ }^{1}$ School of Computer Engineering, Huaiyin Institute of Technology, Huaian, Jiangsu 223003, China \\ ${ }^{2}$ Key Lab of National Mobile Communications, Southeast University, Nanjing, Jiangsu 210096, China \\ ${ }^{3}$ School of Electronic and Electrical Engineering, Huaiyin Institute of Technology, Huaian, Jiangsu 223003, China \\ ${ }^{4}$ National Mobile Communications Research Lab, Southeast University, Nanjing, Jiangsu 210096, China \\ ${ }^{5}$ School of Civil Engineering, Harbin Institute of Technology, Heilongjiang 150000, China
}

Correspondence should be addressed to Jianyang Zhao; jszhaojy@163.com

Received 9 November 2014; Accepted 26 November 2014

Academic Editor: Zidong Wang

Copyright (C) 2015 Jianyang Zhao et al. This is an open access article distributed under the Creative Commons Attribution License, which permits unrestricted use, distribution, and reproduction in any medium, provided the original work is properly cited.

\begin{abstract}
The mobile terminals must be compensated for the Doppler effect in their moving communication. This special characteristic of mobile communication can be developed in some new applications. This paper proposes methods to realize mobile navigation calculation via Doppler shifts. It gives the theory of relationship between the motion parameters, like directions and speed, and frequency shifts caused by multibase stations. The simulation illustrates how to compute the movement parameters of numerical calculation and what should be care for the problem near angle 90 degree. It also gives an application with Google map and dynamical locating position and direction on a mobile phone by public wireless network. Given the simulation analysis and navigation test, the results show that this method has a good effect.
\end{abstract}

\section{Introduction}

The problem of Doppler shift should have its dualities. On the one hand, mobile terminal needs to solve the problem of frequency shift to realize the communication. Many literatures have proposed different calculation methods about frequency shift in mobile communication. Hua Jingyu and You Xiaohu who are in the National Key Laboratory studying about mobile communication in Southeast University utilize a frequency shift calculation system CP-SCBT in [1] by calculating the concomitant difference of time sequence and an algorithm about estimating the square root of the dynamic window oversampling [2]. On the other hand, since the Doppler shift is related to speed and direction [3], it must have value in fields of navigation and positioning. The research of these fields has not been adequately concerned. This paper tries to find the relationship between the motion parameters and frequency shift of multibase stations and then proposes a scheme about mobile terminal autonavigation technology and realization without GPS.

At present, the major global position systems are GPS and AGPS (assisted GPS). The GPS system receives signal from at least three satellites to combine and calculate its own space position. The AGPS is based on network to track record for reducing time cost of position and its dependence on satellites. Sakamoto et al. once proposed TDOA Location method upon multisubstations $[4,5]$. Some scholars [6-8] discuss and illustrate possibilities and fundamental limitations with mobile positioning in available wireless network measurements. System-specific, low-layer techniques for how these are computed for second generation $(2 \mathrm{G})$ and $[9,10]$ for third generation $(3 \mathrm{G})$ can be found, respectively.

However, they have not provided navigation parameters like speed, direction, and so forth. Mobile system gets navigation with the best receptivity for the reason that mobile terminals have provided the real-time estimate of Doppler 
shift in moving process and uses it to optimize the system parameter. Hence, the system is applied to frequency channel measurement, resource allocation, handoff decision power control, and so on [11-13]. The research of Doppler shift navigation not only gets a good use of mobile terminal frequency shift estimation and some correlation properties but also uses $3 \mathrm{G} / 4 \mathrm{G}$ mobile communication system to constitute the mobile navigation system.

\section{Theory and Calculation of Mobile Navigation}

Mobile terminal must estimate Doppler shift which reflects the speed of the channel parameter change. When the moving of terminal is quicker, the Doppler shift is bigger. The mobile terminal can automatically compensate the change of parameter coursed by frequency shift.

2.1. Relationship between Doppler Shift and Parameters. The purpose of researching the relationship between Doppler shift and position is to apply the result of frequency shift to estimate navigation and position of mobile terminal. The method is integrated estimating parameters of position, speed, and direction by different frequency shift of several adjacent base stations to solve the problem of mobile terminal continuous dynamic position.

It is shown as in Figure 1 that frequency shift, transmitting frequency, speed, and direction have the following relationship known from Doppler effect:

$$
f_{d}=\frac{f}{C} v \cdot \cos \alpha
$$

In (1), $\alpha$ is the angle between the moving direction of terminal and spreading direction of signal, $v$ is the moving direction of terminal, $C$ is spreading direction of electromagnetic wave, and $f$ is frequency of carrier.

When the user mobile terminal UE moves in the direction of $\mathrm{O}_{2} \mathrm{O}_{1}$ at speed of $v$, the frequency shifts $f_{d 1}, f_{d 2}$ related to base stations Nodes $B_{1}$ and $B_{2}$ are produced as in the following equations:

$$
\begin{aligned}
& f_{d 1}=\frac{f}{C} v \cdot \cos \alpha_{1}, \\
& f_{d 2}=\frac{f}{C} v \cdot \cos \alpha_{2} .
\end{aligned}
$$

The distance of two base stations is $S$. The distance between user mobile terminal and base stations Node $B_{1}$ and Node $B_{2}$ can be tested as $L_{1}$ and $L_{2}$ via the phase difference of mobile terminal pilot frequency yards. The following formula can be derived by triangle:

$$
\cos \left(\alpha_{2}-\alpha_{1}\right)=\frac{L_{1}^{2}+L_{2}^{2}-S^{2}}{2 L_{1} L_{2}} .
$$

In theory, the frequency shifts $f_{d 1}, f_{d 2}$ can be measured and the speed $v$ and direction angles $\alpha_{1}, \alpha_{2}$ can be calculated by (4). As a result, the position and direction location is realized:

$$
\begin{gathered}
f_{d 1}=\frac{f}{C} v \cdot \cos \alpha_{1}, \\
f_{d 2}=\frac{f}{C} v \cdot \cos \alpha_{2}, \\
\cos \left(\alpha_{2}-\alpha_{1}\right)=\frac{L_{1}^{2}+L_{2}^{2}-S^{2}}{2 L_{1} L_{2}} .
\end{gathered}
$$

In fact, the calculating process is more complex than that mentioned above. The reason is that (4) is a transcendental equation. It needs appropriate transform and the arithmetic solution can be obtained by iterative methods.

Let

$$
\Delta=\alpha_{2}-\alpha_{1}=\cos ^{-1}\left(\frac{L_{1}^{2}+L_{2}^{2}-S^{2}}{2 L_{1} L_{2}}\right) .
$$

The following formula can be achieved by simultaneous equations (4) and (5):

$$
\begin{aligned}
\cos \alpha_{1} \cos \alpha_{2} & =\frac{1}{2}\left(\cos \left(\alpha_{2}+\alpha_{1}\right)+\cos \left(\alpha_{2}-\alpha_{1}\right)\right) \\
& =\left(\frac{C}{f v}\right)^{2} \cdot f_{d 1} \cdot f_{d 2}
\end{aligned}
$$

or

$$
\begin{aligned}
\cos \left(2 \alpha_{2}-\Delta\right)+\cos \Delta & =2\left(\frac{C}{f v}\right)^{2} \cdot f_{d 2} \cdot f_{d 1} \\
& =2\left(\frac{C}{f v} \cdot f_{d 2}\right)^{2} \cdot \frac{f_{d 1}}{f_{d 2}} \\
& =2 \cos ^{2} \alpha_{2} \cdot \frac{f_{d 1}}{f_{d 2}} \\
& =\left(\cos 2 \alpha_{2}+1\right) \cdot \frac{f_{d 1}}{f_{d 2}}
\end{aligned}
$$

Then

$$
\begin{gathered}
\cos \left(2 \alpha_{2}-\Delta\right)+\cos \Delta=\left(\cos 2 \alpha_{2}+1\right) \cdot \frac{f_{d 1}}{f_{d 2}} \\
\cos 2 \alpha_{2} \cdot f_{d 1}-\cos \left(2 \alpha_{2}-\Delta\right) \cdot f_{d 2}=f_{d 2} \cdot \cos \Delta-f_{d 1}
\end{gathered}
$$

It just needs to select different values of the formula $\alpha_{2} \in[0,180]$ in the order of the smallest to the largest and then calculate the value of the two ends. When the different value reaches the required error, the value is the solution as (9). Then all will be calculated when substituting $\alpha_{2}$ to formula (3). The speed $v$ and direction angles $\alpha_{1}, \alpha_{2}$ will be calculated through (2). The moving parameter can be expressed according to $M\left(v, \alpha_{1}, \alpha_{2}\right)$. 


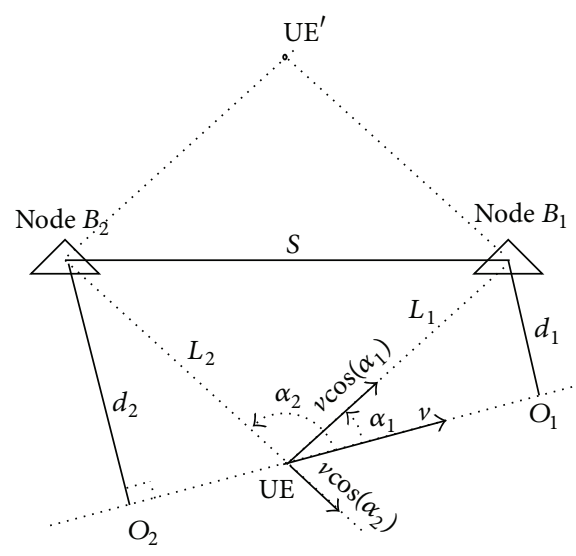

FIGURE 1: Relationship between mobile terminal and base station.

\subsection{The Parameter Calculation Steps of Mobile Terminal Navigation and Position}

Step 1. Receive the carrier frequency signals of the two base stations through antenna unit and then calculate frequency shifts $f_{d 1}, f_{d 2}$. At last, calculate $\alpha_{1}, \alpha_{2}$ through formula (2).

Step 2. If it receives signal from only one base station, it needs to test frequency shift $f_{d 1}\left(\right.$ or $\left.f_{d 2}\right)$ to confirm $\alpha_{1}\left(\right.$ or $\left.\alpha_{2}\right)$. And then, using equations (2) and (9), calculate the $f_{d 2}\left(\right.$ or $\left.f_{d 1}\right)$ of the neighboring base station and $\alpha_{2}$ (or $\left.\alpha_{1}\right)$.

Step 3. Calculate $L_{1}-L_{2}$ by measuring cost time from stations to moving terminal and then calculate the $L_{1}, L_{2}$ with (5).

Step 4. Calculate speed $v$ and direction angles $\alpha_{1}, \alpha_{2}$. The relative position of the mobile terminal $p(x, y)$ can be calculated when using $M\left(v, \alpha_{1}, \alpha_{2}\right)$ expressing the moving parameter. If $B_{1}\left(x_{1}, y_{1}\right), B_{2}\left(x_{2}, y_{2}\right)$ are known, then

$$
\begin{aligned}
& L_{1}^{2}-\left(x-x_{1}\right)^{2}-\left(y-y_{1}\right)^{2}=0, \\
& L_{2}^{2}-\left(x-x_{2}\right)^{2}-\left(y-y_{2}\right)^{2}=0 .
\end{aligned}
$$

It is expressed in parameter form as follows:

$$
p(x, y)=f\left(B_{1}, B_{2}, L_{1}, L_{2}\right) .
$$

\section{Simulation Analysis and Navigation Test}

3.1. Case I: Navigation Parameters Calculating. If $S=5 \mathrm{~km}$, $L_{1}=4 \mathrm{~km}, L_{2}=3 \mathrm{~km}, f_{d 1}=104 \mathrm{~Hz}$, and $f_{d 2}=-38 \mathrm{~Hz}$, simulation calculation will get the following:

$$
\begin{aligned}
& v=59.9773 \mathrm{~km} / \mathrm{h}, \alpha_{2}=110.0079 \text { degrees, and } \alpha_{1}= \\
& 20.0079 \text { degrees, } \\
& \text { or } v=1.7049 e+004, \alpha_{2}=90.0117 \text {, and } \alpha_{1}=0.0117 \text {. }
\end{aligned}
$$

The second solution speed above is out of reality obviously. The reason is that there is a critical problem when $\alpha_{2}$ is close to 90 degrees. The change of $f 1$ is so quick that tiny calculation error will cause a big error of the speed calculation while $\alpha_{2}$ is near 90 degrees.

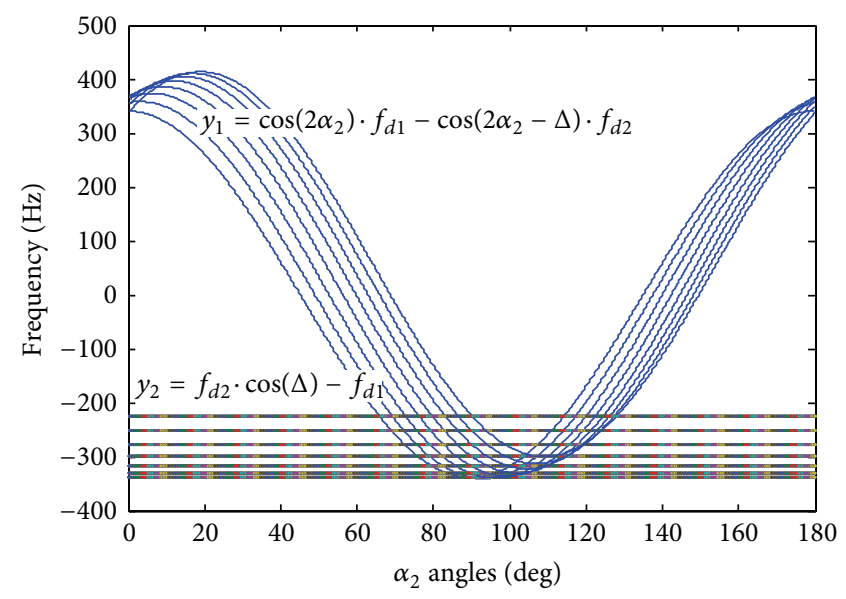

FIgURE 2: Relationship between direction angle and frequency shift in the moving process.

TABLE 1: Data range of direction angles and frequency shifts.

\begin{tabular}{lcccc}
\hline$\alpha_{1}$ & $f_{d 1}$ & $\alpha_{2}$ & $f_{d 2}$ & $O_{2} O_{1}$ direction \\
\hline$[\mathbf{0}, \mathbf{9 0}]$ & $\geq 0$ & {$[0 \sim 90]$} & $\geq 0$ & \\
$(\mathbf{9 0}, \mathbf{1 8 0}]$ & $<0$ & {$[90 \sim 180]$} & $<0$ & Synchrony \\
\hline$[\mathbf{0} \sim \mathbf{9 0}]$ & $\geq 0$ & {$[0,90]$} & $\geq 0$ & \\
$(\mathbf{9 0} \sim \mathbf{1 8 0}]$ & $<0$ & & & Reverse \\
{$[\mathbf{9 0} \sim \mathbf{1 8 0}]$} & $<\mathbf{0}$ & $\mathbf{( 9 0 , \mathbf { 1 8 0 } ]}$ & $<\mathbf{0}$ & \\
\hline
\end{tabular}

3.2. Case II: Navigation Parameters Calculating. If UE as MT (mobile terminal) moves in the $\mathrm{O}_{2} \mathrm{O}_{1}$ direction, the solution $\alpha_{2}$, one of the MT moving parameters $\alpha_{2}$ will be the point of intersection. Distance of UE to base stations Node $B_{1}$ and Node $B_{2}$ is that $d_{1}=2, d_{2}=4$. As shown in Figure 2 , there are two points of intersection of line $y_{1}$ and line $y_{2}$. One is close to 90 degrees and the other is the solution we require. The test process needs to consider this situation. The methods of continuous forward and backward ruin are needed to solve this problem. The detailed discussion is shown as follows.

Direction angles $\alpha_{1}$ and $\alpha_{2}$ of the equation solution may have two solutions. At the time, we need to use the positive or negative of the frequency as judgment. The total possible conditions are shown in Table 1. When $\alpha_{2}$ is close to 90 degrees, the arithmetic of example will be replaced by curve ruin method to get the approximate solution.

3.3. Test of Navigation System. As shown in Figure 3, Huaihai South Road from $(33.4980,119.0258)$ to $(33.5646,119.0323)$ is selected for testing. The distance between base stations $B_{1}$ and $B_{2}$ is $3.38208 \mathrm{~km}$. When the car with test terminal reaches point $M$, the calculation has been finished and shown as follows:

$$
\begin{gathered}
v=60 \mathrm{~km} / \mathrm{h} \\
\alpha=-8.6
\end{gathered}
$$




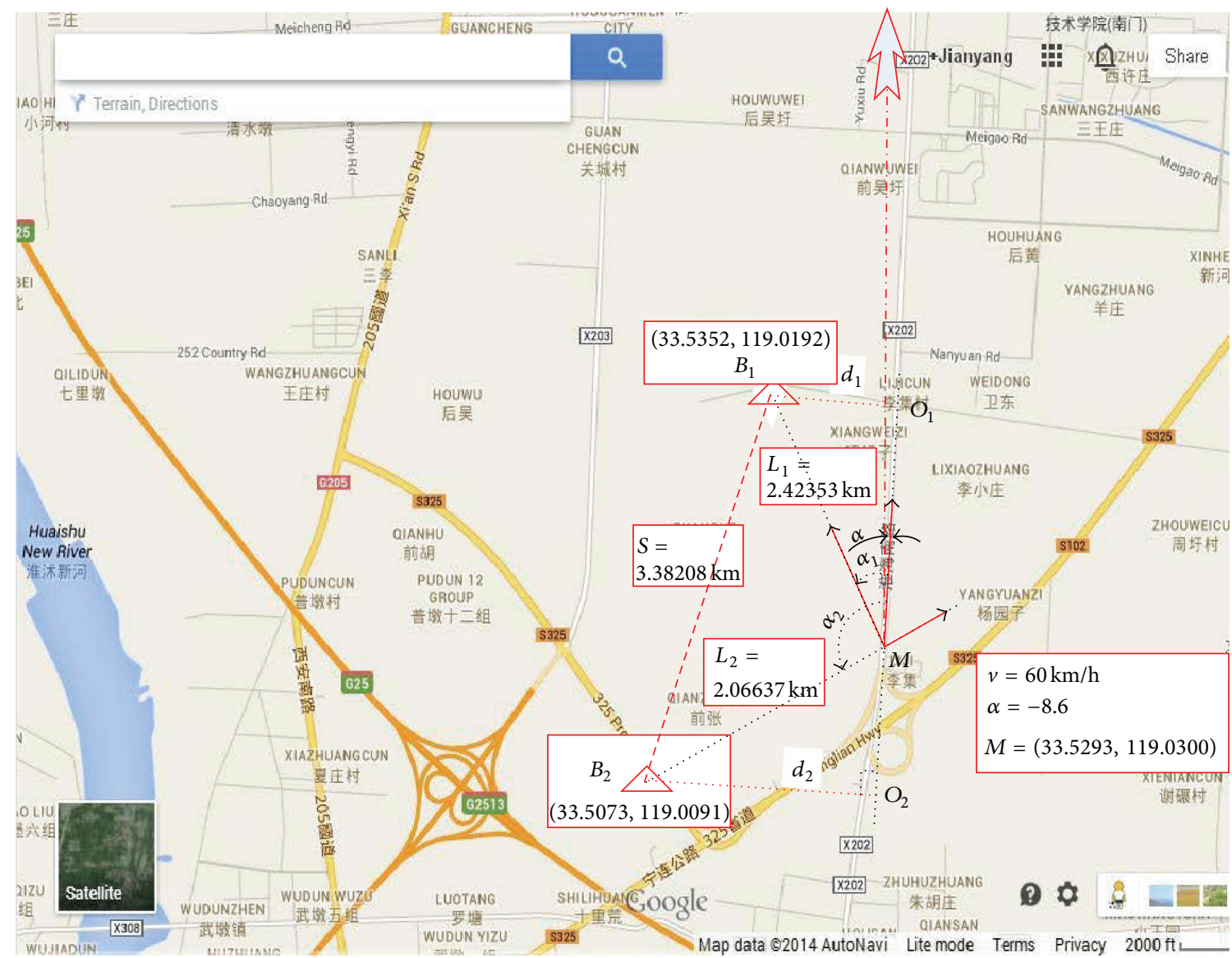

FIGURE 3: In-vehicle navigation showing with position verification.

The real speed is $60.8 \mathrm{~km} / \mathrm{h}$. The traffic map and navigation marks are produced by calculation. The test map comes from Google. Before the system running, sign four GPS points of the map which is 100 times bigger than the used part. In the test process, select a part map nearing point $M$ and then send it to MT after overlying calculation.

\section{Conclusions}

MT automatically adapts the Doppler effects which can play a role in navigation and positioning. Then the MT gets valueadded functions after adding little resource spending. Theoretical analysis and simulation results show that MT position method is feasible. Compared with GPS position method, the benefit of this strategy is the straightway use of mobile channel to transmit navigation and position information without special GPS receiver or communication network. The strategy of navigation position based on cloud platform is feasible through test checking.

The future work is about combining navigation arithmetic of self-adaption signal in the condition of multiroad pilot.

\section{Conflict of Interests}

The authors declare that there is no conflict of interests regarding the publication of this paper.

\section{Acknowledgments}

This work has been supported by the National High Technology Research and Development Program of China (863 Program) (no. 2008A A01Z205) and the National Natural Science Foundation of China (no. 61171081).

\section{References}

[1] H. Jingyu, Y. Xiaohu, S. Bin, and Y. H. Kim, "A scheme for the Doppler shift estimation despite the power control in mobile communication systems," in Proceedings of the IEEE 59th Vehicular Technology Conference (VTC '04), vol. 1, pp. 284-288, May 2004.

[2] J. A. Jahanshahi, S. A. Ghorashi, and H. Sadreazami, "Jamming detection at base station using fuzzy c-means algorithm," in Proceedings of the International Symposium on Computer Networks and Distributed Systems (CNDS '11), pp. 40-44, Tehran, Iran, February 2011.

[3] M. D. Austin and G. L. Stuber, "Velocity adaptive handoff algorithms for microcellular systems," IEEE Transactions on Vehicular Technology, vol. 43, no. 3, pp. 549-561, 1994.

[4] M. Sakamoto, J. Huoponen, I. Niva et al., "Adaptive channel estimation with velocity estimator for WCDMA reciever," in Proceedings of the IEEE Vehicular Technology Conference, vol. 3, pp. 15-18, 2000. 
[5] S. Yin, G. Wang, and X. Yang, "Robust PLS approach for KPIrelated prediction and diagnosis against outliers and missing data," International Journal of Systems Science, vol. 45, no. 7, pp. 1375-1382, 2014.

[6] F. Gustafsson and F. Gunnarsson, "Mobile positioning using wireless networks: possibilities and fundamental limitations based on available wireless network measurements," IEEE Signal Processing Magazine, vol. 22, no. 4, pp. 41-53, 2005.

[7] A. H. Sayed, A. Tarighat, and N. Khajehnouri, "Network-based wireless location: challenges faced in developing techniques for accurate wireless location information," IEEE Signal Processing Magazine, vol. 22, no. 4, pp. 24-40, 2005.

[8] J. Wang, H. Zhao, Y. Bi, X. Chen, R. Zeng, and S. Shao, "Quadrotor helicopter autonomous navigation based on vanishing point algorithm," Journal of Applied Mathematics, vol. 2014, Article ID 567057, 12 pages, 2014.

[9] Y. Zhao, "Standardization of mobile phone positioning for 3G systems," IEEE Communications Magazine, vol. 40, no. 7, pp. 108-116, 2002.

[10] J. J. Caffery, Wireless Location in CDMA Cellular Radio Systems, Kluwer Academic Publishers, Norwell, Mass, USA, 1999.

[11] S. Catreux, V. Erceg, D. Gesbert, and R. W. Heath Jr., "Adaptive modulation and MIMO coding for broadband wireless data networks," IEEE Communications Magazine, vol. 40, no. 6, pp. 108-115, 2002.

[12] S. Yin and Z. Huang, "Performance monitoring for vehicle suspension system via fuzzy positivistic C-means clustering based on accelerometer measurements," IEEE/ASME Transactions on Mechatronics, vol. PP, no. 99, pp. 1-8, 2014.

[13] S. Yin, X. Zhu, and O. Kaynak, "Improved PLS focused on key performance indictor related fault diagnosis," IEEE Transactions on Industrial Electronics, no. 99, 2014. 


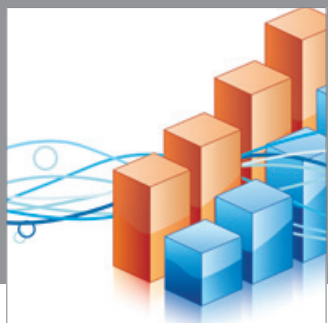

Advances in

Operations Research

mansans

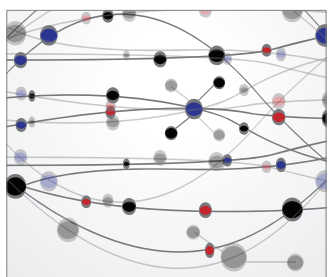

The Scientific World Journal
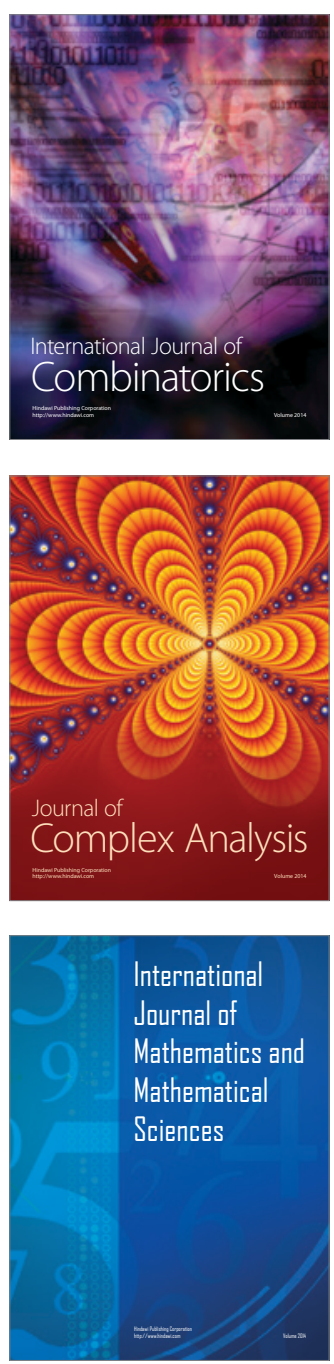
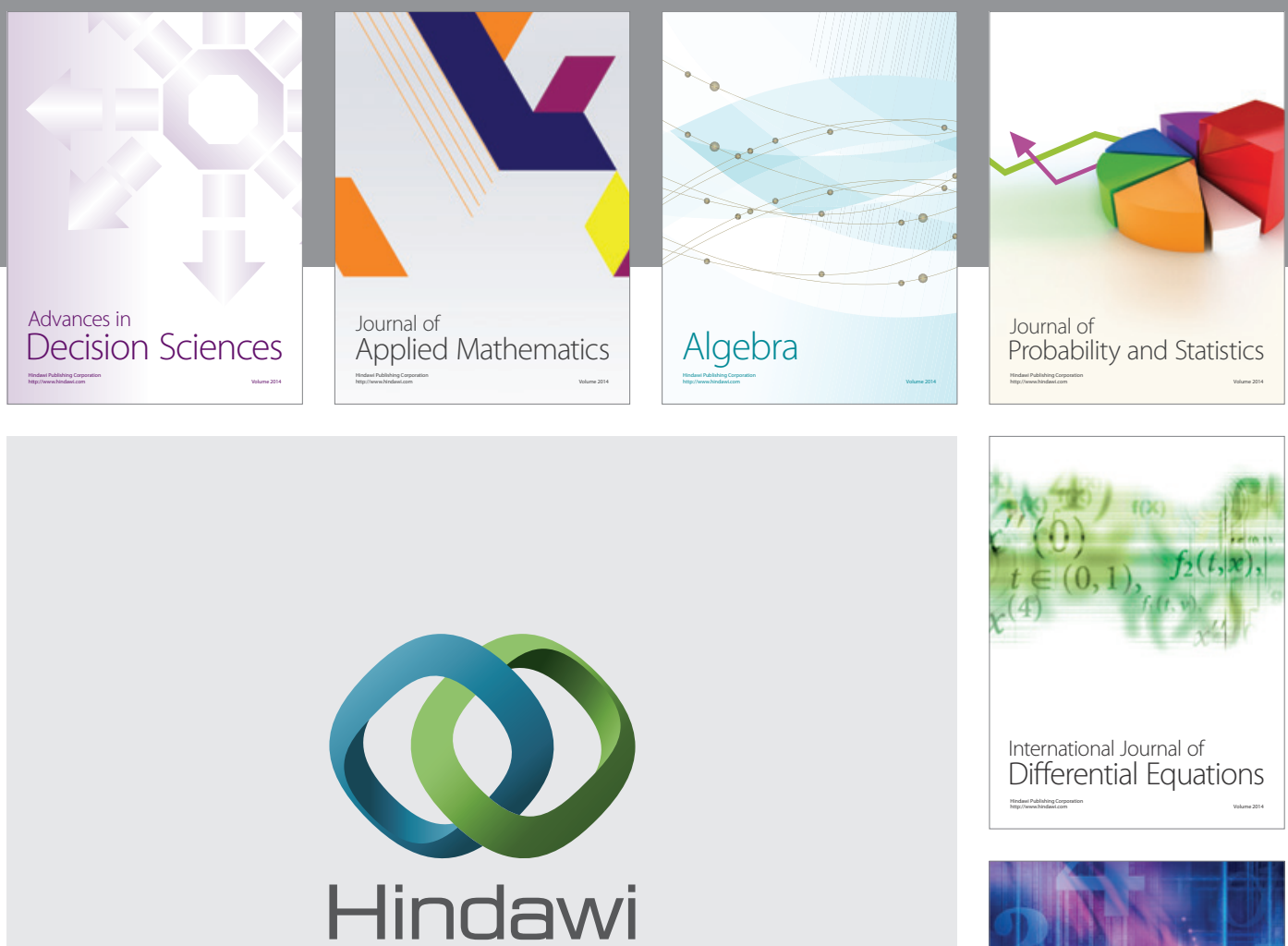

Submit your manuscripts at http://www.hindawi.com
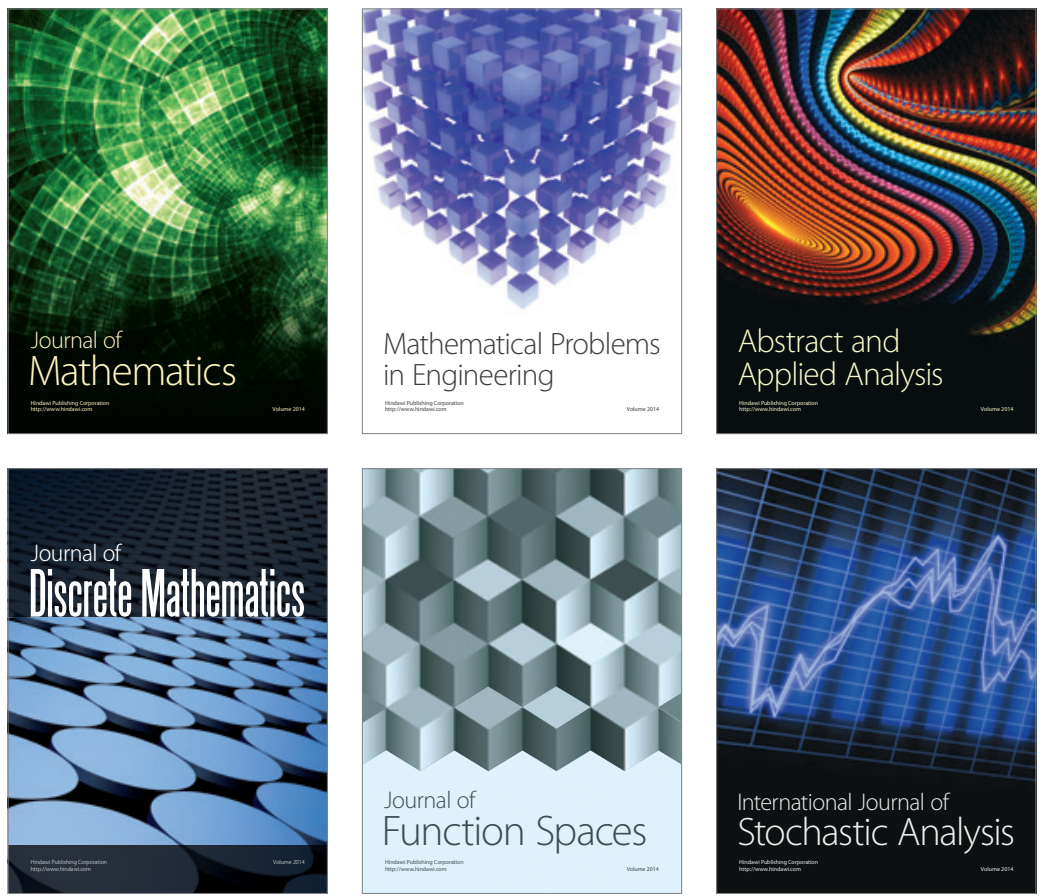

Journal of

Function Spaces

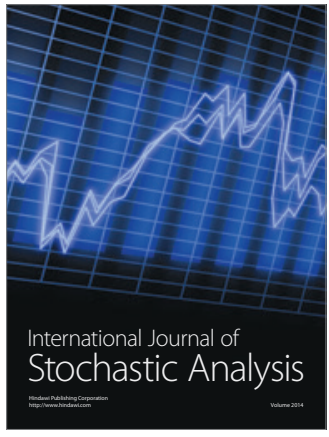

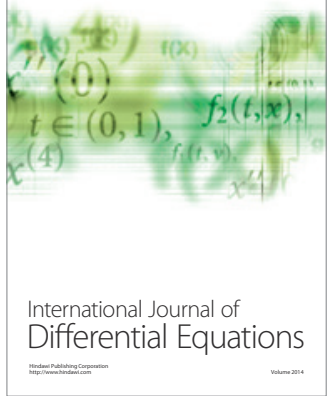
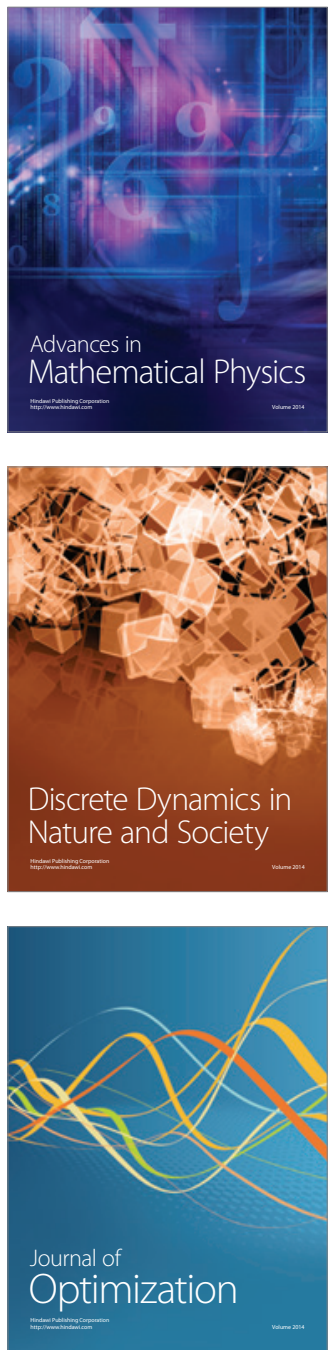